Z Gerontol Geriat 2018 $51: 275-281$ DOI 10.1007/s00391-016-1177-z Eingegangen: 12 . September 2016 Überarbeitet: 29. September 2016 Angenommen: 16. Dezember 2016 Online publiziert: 16. Januar 2017 (c) Der/die Autor(en) 2017. Dieser Artikel ist eine Open-Access-Publikation.

\section{CrossMark}

\author{
S. Krupp ${ }^{1}$ D $\cdot$ A. Seebens ${ }^{1,2,3} \cdot$ J. Kasper ${ }^{1} \cdot$ M. Willkomm ${ }^{1} \cdot$ F. Balck $^{1,2}$ \\ 'Forschungsgruppe Geriatrie Lübeck, Krankenhaus Rotes Kreuz Lübeck - Geriatriezentrum, Lübeck, \\ Deutschland \\ ${ }_{2}^{2}$ Psychosoziale Medizin und Entwicklungsneurowissenschaften, Universitätsklinikum Carl Gustav Carus, \\ Dresden, Deutschland \\ ${ }^{3}$ Universität zu Lübeck, Lübeck, Deutschland
}

\title{
Validierung der deutschen Fassung des Six-Item Screeners
}

\section{Kognitiver Kurztest mit breiten Anwendungsmöglichkeiten}

\begin{abstract}
Der Six-Item Screener (SIS) ist ein etwa einminütiger Kognitionstest ohne Materialeinsatz. Auch die geringe Patientenbelastung macht den Test attraktiv für den Einsatz in der stationären Aufnahme geriatrischer Patienten. Die in der Forschungsgruppe Geriatrie Lübeck 2012 entwickelte, kulturell adaptierte Version ist die erste von Callahan autorisierte deutsche Übersetzung. Ziel der vorliegenden Studie ist die Bestimmung der Gütekriterien Reliabilität, Validität und Änderungssensitivität des SIS als Instrument des geriatrischen Assessments zur Erfassung kognitiver Störungen.
\end{abstract}

\section{Grundlagen}

Der SIS wurde von Callahan et al. [6] eingeführt, um in der Rekrutierungsphase von Studien ggf. kognitive Störungen als Ausschlusskriterium zu erfassen. Er ist auch telefonisch durchführbar. Die Auswertung erfolgt mithilfe der Summierung der Anzahl korrekter Antworten. Die Autoren empfehlen die Anpassung des Grenzwerts an die Intention der Testung.

Callahan et al. validierten den SIS an 2 Stichproben: 344 Afroamerikanern aus der Allgemeinbevölkerung (Alter 65 bis 99 Jahre, Mittelwert 74,4 Jahre, 59,4\% Frauen, $4,3 \%$ mit Demenz im Sinne alltagsrelevanter kognitiver Störung) und
651 Patienten eines Alzheimer-Zentrums (Alter 21 bis 92 Jahre, Mittelwert 69,6 Jahre, 57,1\% Frauen, 53,0 \% mit Demenz). Mit der klinischen Diagnose als Referenzstandard ergaben sich bei Unterschreiten von 4 Punkten (5 Punkten) für die Diagnose einer Demenz eine Sensitivität von $88,7 \%(96,8 \%)$ und eine Spezifität von $88,0 \%(68,6 \%)$ in der Allgemeinbevölkerung bzw. von $80,6 \%(89,6 \%)$ und 90,9 \% (79,4\%) im Alzheimer-Zentrum. In anderen Studien $[7,14,20]$ wurden z. T. stark abweichende Ergebnisse gefunden; die Vergleichbarkeit war durch die Wahl anderer Grenzwerte oder Referenztests oder einer differenten Zusammensetzung der Studienpopulation reduziert.

Die von Callahan verwendeten Items wurden in einer "Item-response“-Analyse der bekannteren Mini-Mental State Examination (MMSE, [9, 11]) als die sensitivsten für die Erkennung von Frühsymptomen einer Alzheimer-Demenz ermittelt [8]. Sie schlugen bis zu 4 Jahre vor klinischem Krankheitsbeginn an [2]. Die Inhaltsvalidität ist hierdurch für den SIS belegt. Aufgrund der simplen Durchführung und Auswertung ist der Test für Untersucher leicht erlernbar und eine höhere Objektivität auszunehmen als bei der deutlich untersucherabhängigen MMSE $[5,21]$.

\section{Studiendesign und Unter- suchungsmethoden \\ Studiendesign}

In die monozentrische, diagnostische prospektive Kohortenstudie wurden gemäß positivem Votum der Ethikkommission der Medizinischen Fakultät Dresden (EK 113032015) alle in der Rekrutierungsphase im Krankenhaus Rotes Kreuz Lübeck - Geriatriezentrum konsekutiv stationär aufgenommenen Patienten inkludiert, bei denen keine Ausschlusskriterien (mangelnde Kommunikationsfähigkeit gemäß dem aufnehmenden Arzt, fehlende schriftliche Einwilligung) vorlagen. Als Referenzstandard zur Bestimmung der Sensitivität und der Spezifität diente das klinische Arzturteil.

Für die konvergente Validität wurden die MMSE, der Uhrentest nach Shulman („clock-drawing test“, CDT) $[18,19]$ und Untertests des Regensburger Wortflüssigkeitstests (RWT, [1]) eingesetzt, für die diskriminante Validität die Montgomery-Åsberg Depression Rating Scale (MADRS, [13]).

Der erste SIS ( SIS $_{1}$ ) wurde im Rahmen der Aufnahmeuntersuchung vom Arzt durchgeführt, alle weiteren Kognitionstests von einer Doktorandin. An Tag 3 erfolgten $\mathrm{SIS}_{2}$ und $\mathrm{RWT}_{1}$, an Tag $4 \mathrm{MMSE}_{1}$ und $\mathrm{CDT}_{1}$, an Tag $5 \mathrm{SIS}_{3}$ und randomisiert an Tag $15 \mathrm{SIS}_{4}$ und $\mathrm{RWT}_{2}$, an Tag 16 $\mathrm{MMSE}_{2}$ und $\mathrm{CDT}_{2}$ oder in entgegenge- 


$\begin{array}{ll}\text { Tag 1 } & \rightarrow n=246 \\ \text { Tag 3 } & \rightarrow n=165 \\ \text { Tag 4 } & \rightarrow n=157 \\ \text { Tag 5 } & \rightarrow n=155 \\ \text { Tag 15 } & \rightarrow n=138 \\ \text { Tag 16 } & \rightarrow n=136\end{array}$

Abb. 1 ॥ Zeitliche Verteilung der Durchführung kognitiver Tests

\begin{abstract}
Ich nenne Ihnen 3 Dinge. Bitte warten Sie, bis ich alle 3 Wörter gesagt habe, und wiederholen Sie sie dann. Merken Sie sich die Wörter, denn ich frage Sie demnächst noch einmal danach. Bitte wiederholen Sie:

(1. Wort) - (2. Wort) - (3. Wort) z.B. "Auto - Blume - Ball“

(Maximal 3 Lernversuche $\rightarrow$ alle 3 Wörter richtig wiederholt?

$\square$ ja $\square$ nein)
\end{abstract}

\section{Jetzt stelle ich Ihnen 3 Fragen: \\ Welches Jahr haben wir? \\ Welchen Monat haben wir? \\ Welchen Wochentag haben wir?

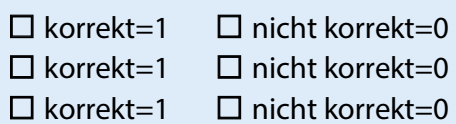

\section{Wie hießen die 3 Dinge, die Sie sich merken sollten?}

\begin{tabular}{|c|c|c|}
\hline rt) & $\square$ korrekt=1 & $\square$ nicht korrekt $=0$ \\
\hline & $\square$ & $\mathrm{xt}=0$ \\
\hline & $\square$ & [ \\
\hline
\end{tabular}

Anzahl der erreichten Punkte: 16

Abb. 2 \ Durchführung des Six-Item Screeners

setzter Reihenfolge (• Abb. 1). Für die Validität wurde $\mathrm{SIS}_{1}$ betrachtet, für die Reliabilität SIS $_{2}$ mit SIS $_{1}$ und SIS $_{3}$ verglichen, für die Änderungssensitivität der Verlauf von MMSE und CDT mit SIS $_{1}$ und $\mathrm{SIS}_{4}$. Das Ergebnis der MADRS wurde der Patientenakte entnommen.

Für die statistische Auswertung wurde das Signifikanzniveau $\alpha \leq 0,05$ gewählt. Die Analysen wurden mithilfe von IBM SPSS Statistics 22 durchgeführt.

\section{Referenzstandard Arzturteil}

Als Referenzstandard für die Diagnose einer kognitiven Störung wurde das klinische Arzturteil festgelegt. Während der behandelnde Arzt aus ethischen Gründen hinsichtlich des kognitiven Assess- me, Störungen des Tag-Nacht-Rhythmus und andere Verhaltensauffälligkeiten.

Es erfolgte eine 3-stufige Einordnung in "kein Hinweis auf kognitive Störung“, "Verdacht auf/leichte kognitive Störung“ und "alltagsrelevante kognitive Störung“ (z. B. Demenz).

\section{Six-Item Screener}

Der SIS (• Abb. 2) hängt von verbalem Verständnis, Konzentration, zeitlicher Orientierung und der Fähigkeit zum verzögerten Abruf von 3 Objekten ab. Diese werden genannt (im Original: „apple - table - penny“, entsprechend Apfel - Tisch - Pfennig oder Cent) und sollen sofort wiederholt werden zur Kontrolle, ob Hören und sprachliche Fähigkeiten als Voraussetzung für das erfolgreiche Reproduzieren ausreichen. Dafür sind maximal 3 Lernversuche erlaubt. Es folgen 3 Fragen zur zeitlichen Orientierung, die gleichzeitig der Distraktion dienen. Für die korrekte Nennung von Wochentag, Monat, Jahr und jeden der danach noch erinnerten 3 Begriffe wird je ein Punkt vergeben. Dabei ist die Reihenfolge der Nennung beliebig.

$\mathrm{Da}$ der SIS insgesamt 4-mal durchgeführt wurde $\left(\mathrm{SIS}_{1}-\mathrm{SIS}_{4}\right)$ und mögliche Lerneffekte von SIS zu SIS und SIS zu MMSE vermieden werden sollten, wurden statt des von uns bereits für alterstraumatologische Patienten eingeführten Worttripletts „Auto - Blume - Ball“ 4 andere aus dem Grundwortschatz der 500 häufigsten Wörter zusammengestellt, die jeweils 2 zweisilbige Objekte (keine abstrakten Begriffe) und ein einsilbiges bezeichnen. Callahan ist mit dieser Anpassung einverstanden. Die Worttripletts wurden für jeden Probanden randomisiert auf die verschiedenen Durchführungszeitpunkte verteilt.

\section{Mini-Mental State Examination}

Die Mini-Mental State Examination (MMSE; [9, 11]) berücksichtigt außer den für den SIS genannten Faktoren auch räumliche Orientierung, Merkfähigkeit, Rechnen/Buchstabieren, Lesen, Benennen und Visuokonstruktion. $\mathrm{Zu}$ den Schweregraden kognitiver Defizite korrespondierende Grenzwerte sind 
Z Gerontol Geriat 2018·51:275-281 ～DOI 10.1007/s00391-016-1177-z

(c) Der/die Autor(en) 2017. Dieser Artikel ist eine Open-Access-Publikation.

S. Krupp · A. Seebens · J. Kasper · M. Willkomm · F. Balck

\section{Validierung der deutschen Fassung des Six-Item Screeners. Kognitiver Kurztest mit breiten Anwendungsmöglichkeiten}

\section{Zusammenfassung}

Hintergrund. Besonders in der Aufnahmesituation soll die Erfassung alltagsrelevanter kognitiver Defizite Patient und Untersucher möglichst wenig belasten. Der Six-Item Screener (SIS) dauert etwa 1 min, ist leicht erlernbar, erfordert kein Material und ist unabhängig von den Seh- und feinmotorischen Fähigkeiten des Patienten. Die Erstveröffentlichung erfolgte 2002 durch Callahan, der die vorliegende deutsche Übersetzung genehmigte.

Ziel der Arbeit. Wir überprüften die konvergente und diskriminante Validität, Reliabilität und Änderungssensitivität der deutschen Übersetzung bei geriatrischen Patienten. Material und Methoden. Es absolvierten 165 Patienten der Akutgeriatrie in den ersten
16 Tagen: SIS (4-mal), Mini-Mental State Examination (MMSE, 2-mal), Uhrentest nach Shulman (2-mal), Regensburger Wortflüssigkeitstest (2-mal) und Montgomery-Åsberg Depression Rating Scale. Als Referenzstandard diente das Gesamturteil eines in Bezug auf die Testergebnisse verblindeten Arztes. Ergebnisse. Der SIS korrelierte eng mit dem ärztlichen Urteil $(-0,729)$. Die RetestReliabilität lag bei 0,705 , die interne Konsistenz bei 0,821 (Cronbachs a). Die Sensitivität für das Erfassen alltagsrelevanter kognitiver Störungen betrug $100 \%$, wenn ein Grenzwert von 5 Punkten gewählt wurde. Bei voller Punktzahl sind auch leichte kognitive Störungen weitgehend ausgeschlossen.
Schlussfolgerung. Der SIS ist ein valider, reliabler kognitiver Kurztest. Unter Verwendung eines Grenzwerts von 5 erfasst er alltagsrelevante kognitive Defizite mit höherer Sensitivität als die MMSE beim Grenzwert von 25. Erreichen klinisch unauffällige geriatrische Patienten den Maximalwert, besteht keine medizinische Indikation zu weiterem kognitiven Assessment. Kürze und einfache Durchführbarkeit des SIS erlauben seine Anwendung auch außerhalb geriatrischer Stationen.

Schlüsselwörter

Geriatrisches Assessment - Psychometrische Kriterien · Kognition · Demenz $\cdot$ Screening

\section{Validation of the German version of the 6-item screener. Brief cognitive test with broad application possibilities}

\section{Abstract}

Background. Especially during admission the detection of cognitive deficits relevant to everyday life should burden patient and examiner as little as possible. The 6-item screener (SIS) takes approximately $1 \mathrm{~min}$, is easy to learn, does not require any material and is independent of the patient's visual and fine motor skills. The test was first published in 2002 by Callahan who approved the present German translation.

Objective. We checked the convergent and discriminant validity, reliability and sensitivity to change of the German translation among geriatric patients.

Material and methods. A total of 165 patients in an acute geriatric department performed the SIS (4 times), the mini mental state examination (MMSE, 2 times), clock-drawing test according to Shulman ( 2 times), the Regensburg verbal fluency test ( 2 times) and the Montgomery-Åsberg depression rating scale within a period of 16 days. The overall judgment of a physician blinded to the test results served as the reference standard. Results. The SIS closely correlated with the medical judgment $(-0.729)$. The retest reliability was 0.705 and the internal consistency 0.821 (Cronbach's alpha). The sensitivity to detect cognitive deficits relevant to activities of daily living was $100 \%$ if a threshold of 5 points was chosen. The achievement of maximum points largely rules out even mild cognitive impairment.
Conclusion. The SIS is a valid, reliable short cognitive test. Using a threshold of 5 points the SIS detects cognitive deficits relevant to daily living with a higher sensitivity than the MMSE with a threshold of 25 . When the maximum score is achieved there are no medical indications for further cognitive assessment of clinically unremarkable geriatric patients. The brevity and simple application of the SIS also enable its application outside geriatric wards.

\section{Keywords}

Geriatric assessment - Psychometrics .

Cognition · Dementia $\cdot$ Screening umstritten. Aktuell wird mehrheitlich ein Unterschreiten von 25 der maximal 30 Punkte als Hinweis auf eine alltagsrelevante kognitive Störung gewertet. Für die Retest-Reliabilität werden Werte von 0,80 bis 0,95 genannt [15].

\section{Uhrentest nach Shulman}

Beim CDT $[18,19]$ müssen in einen vorgegebenen Kreis die Zahlen des Ziffernblatts eingetragen und die Zeiger für die
Uhrzeit „10 min nach 11 “ eingezeichnet werden. Neben der Fähigkeit, die verbal vermittelte digitale Instruktion in eine analoge zu übersetzen, kommen zeitliches Mengenwissen und visuokonstruktive Fähigkeiten zum Tragen. Bewertet wird das Ergebnis anhand von Beurteilungskriterien mit einem Score zwischen 1 (perfekt) und 6 (keine erkennbare Darstellung einer Uhr); auch Beispielbilder sind verfügbar. Ein Score über 2 wird als Hinweis auf eine Störung der Kognition interpretiert. Shulman nennt eine Interrater-Reliabilität von 0,94 .

\section{Regensburger Wortflüssigkeitstest}

Der Regensburger Wortflüssigkeitstest (RWT, [1]) testet die semantische und die phonetische Wortflüssigkeit. Die Reliabilität variiert je nach Auswahl aus den 10 Untertests. Vier davon wurden durchgeführt. Die Studienteilnehmer sollten jeweils $1 \mathrm{~min}$ lang Wörter mit 


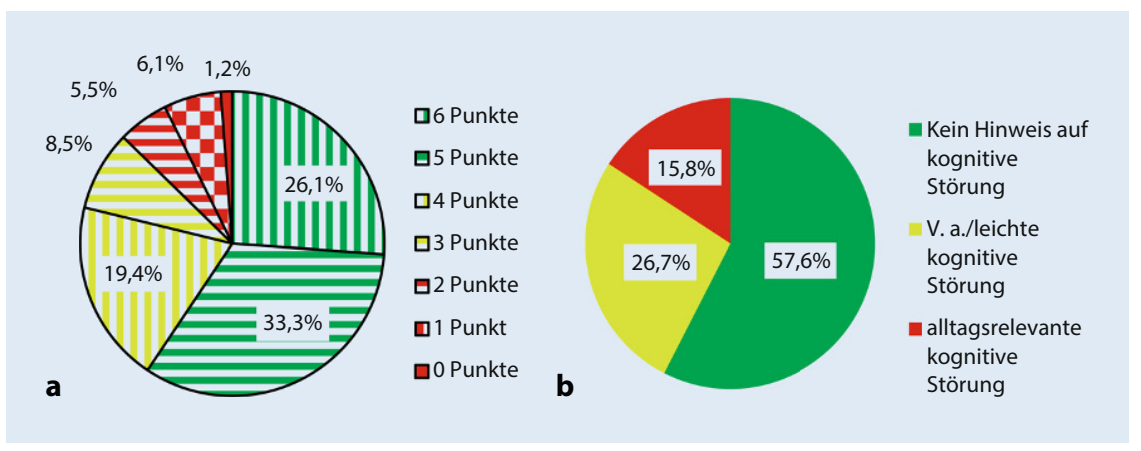

Abb. 3 A Verteilung der Häufigkeiten der Ergebnisse. a Erster Six-Item Screener (SIS 1 , bei Aufnahme), b Arzturteil

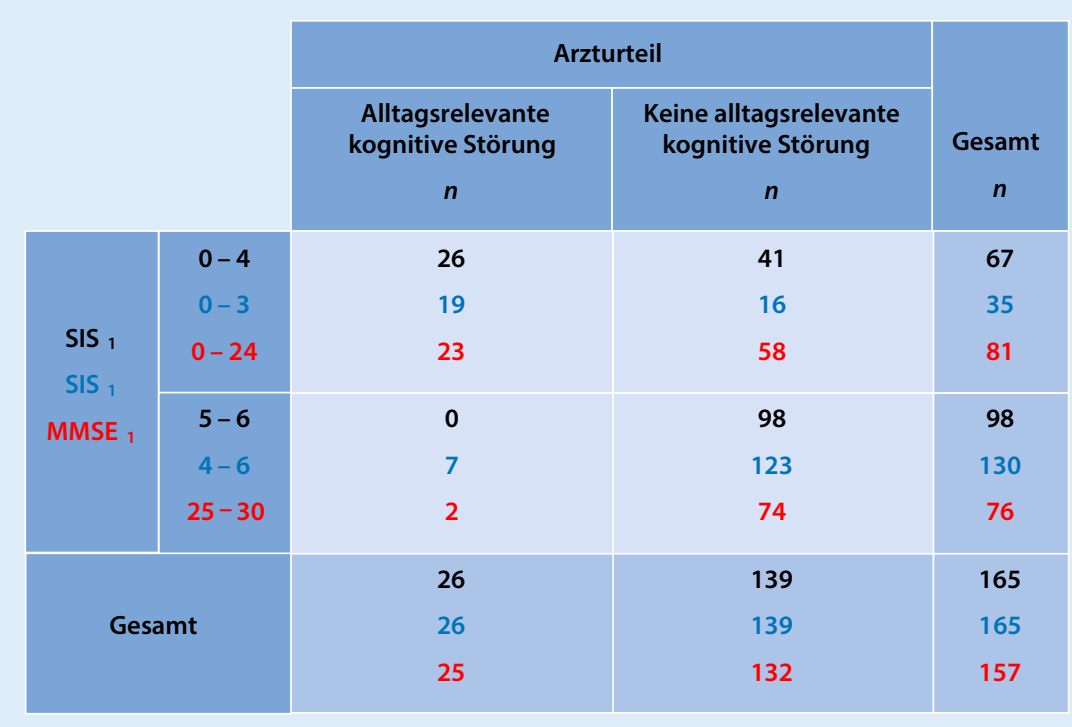

Abb. 4 \ Vierfeldertafel aus erstem Six-Item Screener (SIS 1 ) mit unterschiedlichen Grenzwerten und erster Mini-Mental State Examination $\left(\mathrm{MMSE}_{1}\right)$ /Arzturteil

dem Anfangsbuchstaben „P“ bzw. „M“ oder aus der Kategorie „Tiere“ bzw. „Lebensmittel" nennen. Die Reihenfolge der Aufgaben erfolgte randomisiert. Für die Auswertung wurden die Ergebnisse mit altersnormierten Prozenträngen verglichen und die Mittelwerte der Untertests angegeben. Wir werteten ein Unterschreiten des 16. Prozentrangs als Hinweis auf eine kognitive Störung.

\section{Datengewinnung}

Vom 13.02.2015 bis zum 18.03.2015 wurden 246 Patienten im Krankenhaus Rotes Kreuz Lübeck - Geriatriezentrum stationär aufgenommen. Bei 12 Personen konstatierte der aufnehmende Arzt die fehlende Teilnahmefähigkeit, 51 lehnten die Teilnahme primär oder rückwirkend ab, 8

\section{Ergebnisse}

Es wurden 95 der 165 Studienteilnehmer (57,6 \%) gemäß abschließendem Arzturteil als während der stationären Behandlung durchgehend kognitiv unauffällig eingestuft. Bei 44 Patienten (26,7 \%) wurde eine leichte Störung der Kognition („mild cognitive impairment“, MCI) diagnostiziert, oder es bestand zumindest zeitweise der Verdacht auf eine kognitive Störung. Bei weiteren 26 Patienten $(15,8 \%)$ wurde eine alltagsrelevante Störung der Kognition festgestellt. Somit bestand bei $43 \%$ der Probanden mindestens zeitweise der Verdacht auf eine kognitive Störung. Die Häufigkeitsverteilungen der Messwerte für den SIS bei Aufnahme $\left(\mathrm{SIS}_{1}\right)$ und der Kognition gemäß dem abschließenden Arzturteil sind • Abb. 3 zu entnehmen.

Die Ergebnisse des SIS $_{1}$ waren nicht geschlechtsabhängig $(p=0,924)$. In der univariaten Varianzanalyse mit 4 Altersgruppen entsprechend den Quartilen zeigte sich eine Altersabhängigkeit $(p<0,001)$. Dies lässt sich mit der altersabhängig ansteigenden Prävalenz demenzieller Erkrankungen erklären. In der Untergruppe der gemäß Arzturteil kognitiv Gesunden bestand keine Altersabhängigkeit $(p=0,238)$. Die Ergebnisse beim jeweils erstmaligen Einsatz der dort genannten Instrumente über alle Teilnehmer und in Abhängigkeit vom abschließenden klinischen Arzturteil zeigt • Tab. 1.

Am Aufnahmetag lagen die Ergebnisse geringfügig, aber signifikant $(p=$ $0,026)$ niedriger als bei der folgenden Testung. Der T-Test für verbundene Stichproben ergab keinen signifikanten Unterschied zwischen den Mittelwerten von $\mathrm{SIS}_{2}, \mathrm{SIS}_{3}$ und $\mathrm{SIS}_{4}$ ( $p$ jeweils >0,7; $\mathrm{SIS}_{1}$ $4,43 \pm 1,50 ; \mathrm{SIS}_{2} 4,62 \pm 1,38 ; \mathrm{SIS}_{3} 4,66 \pm$ 1,$48 ;$ SIS $\left._{4} 4,56 \pm 1,46\right)$.

Die Korrelation zwischen SIS $_{1}$ durch den Arzt am Aufnahmetag und $\mathrm{SIS}_{2}$ durch die Doktorandin 2 Tage danach betrug 0,705 ( $p \leq 0,001)$, zwischen $\mathrm{SIS}_{2}$ und $\mathrm{SIS}_{3}$ 0,696 sowie zwischen $\mathrm{SIS}_{3}$ und $\mathrm{SIS}_{4}$ 0,651. Somit lag die Retest-Reliabilität mit und ohne Untersucherwechsel in gleicher Größenordnung, was für hohe Objektivität spricht. Es ergab sich mit 0,821 ein guter Cronbachs- $\alpha$-Wert (zum 
Tab. 1 Ergebnisse der eingesetzten Testverfahren bei der ersten Durchführung in Abhängigkeit von der ärztlichen Einschätzung der Kognition

\begin{tabular}{|c|c|c|c|c|}
\hline \multirow[t]{2}{*}{ Test } & $\begin{array}{l}\text { Mittelwert } \\
\text { über alle Teil- } \\
\text { nehmer }\end{array}$ & $\begin{array}{l}\text { Kein Hinweis } \\
\text { auf kognitive } \\
\text { Störung }\end{array}$ & $\begin{array}{l}\text { MCI/Verdacht } \\
\text { auf kognitive } \\
\text { Störung }\end{array}$ & $\begin{array}{l}\text { Kognitive Stö- } \\
\text { rung mit Alltags- } \\
\text { relevanz }\end{array}$ \\
\hline & $M \pm S D$ & $M \pm S D$ & $M \pm S D$ & $M \pm S D$ \\
\hline $\mathrm{SIS}_{1}$ & $4,43 \pm 1,50$ & $5,20 \pm 0,77$ & $4,16 \pm 1,22$ & $2,08 \pm 1,35$ \\
\hline MMSE $_{1}$ & $23,12 \pm 5,26$ & $25,86 \pm 2,84$ & $22,07 \pm 3,58$ & $15,24 \pm 5,78$ \\
\hline $\mathrm{CDT}_{1}$ & $3,12 \pm 1,23$ & $2,64 \pm 1,18$ & $3,39 \pm 0,92$ & $4,36 \pm 0,81$ \\
\hline $\begin{array}{l}\mathrm{RWT}_{1} \\
\text { (Prozentrang) }\end{array}$ & $26,34 \pm 22,12$ & $34,60 \pm 22,15$ & $20,28 \pm 18,37$ & $6,81 \pm 7,97$ \\
\hline \multicolumn{5}{|c|}{$\begin{array}{l}C D T \text { "clock-drawing test" (Uhrentest nach Shulman), } M \text { Mittelwert, } M C I \text { "mild cognitive impair- } \\
\text { ment" (leichte Störung der Kognition), MMSE Mini-Mental State Examination, RWT Regensburger } \\
\text { Wortflüssigkeitstest, SD Standardabweichung, SIS Six-Item Screener }\end{array}$} \\
\hline
\end{tabular}

Tab. 2 Validität des Six-Item-Scores (SIS) bei alternativen Grenzwerten und der Mini-Mental State Examination (MMSE) für die Erfassung alltagsrelevanter kognitiver Störung, Korrelation mit dem Arzturteil

\begin{tabular}{llll} 
& SIS & SIS $_{\mathbf{1}}$ & MMSE $_{\mathbf{1}}$ \\
\hline Positiv, wenn ... & $<5$ & $<4$ & $<25$ \\
\hline Sensitivität & 1,000 & 0,731 & 0,920 \\
\hline Spezifität & 0,705 & 0,885 & 0,561 \\
\hline Positiver prädiktiver Wert & 0,388 & 0,543 & 0,284 \\
Negativer prädiktiver Wert & 1,000 & 0,946 & 0,974 \\
\hline AUC & 0,937 & & 0,908 \\
\hline Korrelation mit Arzturteil & $-0,729$ & & $-0,715$ \\
\hline AUC ${ }_{\text {"area under the curve" (Fläche unter der Kurve) }}$ & & \\
\hline
\end{tabular}

Vergleich: Cronbachs a bei der MMSE $0,79[4])$.

Für die Änderungssensitivität wurden die Daten jener 13 Patienten gesondert betrachtet, bei denen sich MMSE (um mindestens 3 Punkte) und CDT im Verlauf gleichsinnig entwickelten. Hierbei fand sich ein partielles $\eta^{2}$ von 0,25 als Ausdruck dessen, dass der SIS kognitive Schwankungen, die sich von Tag 3 zu Tag 15/16 sowohl in der MMSE als auch im CDT bemerkbar machten, gut widerspiegeln konnte.

Der SIS korrelierte mit dem Arzturteil $(-0,729)$, gefolgt von der MMSE $(0,677)$, dem CDT $(-0,478)$ und dem RWT $(0,445$; jeweils erste Durchführung). Diese Korrelationen sind auf dem Niveau von 0,01 signifikant, die konvergente Validität ist damit gegeben. Ein für die divergente $V a$ lidität herangezogener Test zur Depressionserfassung (MADRS) korrelierte erwartungsgemäß nicht.

Für die diagnostische und prognostische Kriteriumsvalidität wurde zum einen berechnet, wie zuverlässig und bei
Reduktion der Sensitivität auf $73,1 \%$ (• Abb. 4; - Tab. 2).

Von 70 Patienten, bei denen im klinischen Arzturteil mindestens zeitweise der Verdacht auf eine kognitive Störung bestand, hatten nur 7 im SIS bei Aufnahme die volle Punktzahl erreicht. Bei so streng gewähltem Grenzwert war eine Sensitivität von $90 \%$ für das Erkennen jeglicher im Verlauf auffallender kognitiver Defizite erreicht (einschließlich MCI; NPW 0,84), jedoch ist dann die Spezifität mit $38 \%$ niedrig (PPW 0,52). Probanden mit einem SIS ${ }_{1}$ von 5 (4) erreichten in der MMSE einen arithmetischen Mittelwert von $24,89 \pm 3,32(23,10 \pm 3,67)$, im CDT $2,52 \pm 1,07(3,48 \pm 0,99)$.

\section{Diskussion}

Die logistische Schwierigkeit, rechtzeitig eine schriftliche Einwilligung der Person zu erhalten, die den Patientenwillen vertritt, schlägt sich in der mit 15,8\% relativ niedrigen Quote [17] alltagsrelevant kognitiv beeinträchtigter Studienteilnehmer gemäß klinischem Arzturteil nieder. Es bestätigt sich, dass die Testergebnisse geschlechts- und altersunabhängig interpretiert werden können. Der alterskorrelierte Abfall der erzielten Werte ist über die ansteigende Prävalenz kognitiv gestörter Patienten erklärlich.

Die meisten Probanden zeigten in der stärker stressbelasteten Aufnahmesituation eine signifikant leicht schlechtere Leistung als an den Folgetagen. Da jedoch für den praktischen Nutzen der Anwendung das Ergebnis beim Erstkontakt höhere klinische Relevanz hat, legten wir für unsere Berechnungen zur Validität diesen Wert zugrunde. Auch der große Pool unterschiedlicher Untersucher bei der ersten Erhebung (etwa 20 verschiedene Ärzte) entsprach Alltagsbedingungen.

Bei Einsatz unterschiedlicher Worttripletts wichen die Mittelwerte des SIS an den Tagen 3, 5 und 15/16 nicht signifikant voneinander ab. Somit war auch bei kurzfristiger Wiederholung kein Lerneffekt zu verzeichnen.

In der Geriatrie gehört das Assessment der Kognition zum Standard. Bei einem SIS von 6 Punkten, den in unserer Studie immerhin 26,1\% der Pa- 
tienten erreichten, ist ohne anamnestische oder klinische Auffälligkeiten kein weiteres kognitives Assessment medizinisch erforderlich. Ansonsten sollte der SIS durch ein weiteres Instrument ergänzt werden. Bei 5 oder 4 Punkten sind hierfür Tests zu bevorzugen, die sich zur Frühdiagnostik einer demenziellen Entwicklung eignen; erst unter 4 Punkten im SIS greift die MMSE mit ausreichender Treffsicherheit.

Die Validierung von Tests erfolgt in der Regel an Probanden, die keine gesundheitlichen Einschränkungen jenseits des Gegenstands der Untersuchung aufweisen, die das Testergebnis beeinflussen können. Ältere Patienten leiden jedoch häufig unter sensorischen und feinmotorischen Defiziten sowie stark reduzierter Belastbarkeit. Dies schränkt die Aussagefähigkeit vieler kognitiver Tests ein. Der in $1 \mathrm{~min}$ zu absolvierende, weder Visus noch Handeinsatz benötigende SIS ist dadurch weniger störanfällig als Tests vergleichbarer Aussagekraft, deutlich zeitökonomischer und unterliegt keinem Copyright. Die vorliegende Studie bestätigt ihn in seiner deutschen Version als Kurztest mit guter interner Konsistenz, Kriteriums- und prognostischer Validität sowie zufriedenstellender Reliabilität und Änderungssensitivität bei geriatrischen Krankenhauspatienten.

Untersucher können den SIS leicht erlernen und benötigen kein Material. Die günstige Relation zwischen Aufwand und Aussagekraft machen ihn für den breiten Einsatz auch außerhalb der Geriatrie interessant. So konnte er im Rahmen alterstraumatologischer Kooperation in den zuweisenden Kliniken zur Risikostratifizierung zwecks frühzeitigem Einsatz flankierender Maßnahmen [3] der Delirprävention eingeführt werden. Rundshagen nennt in ihrer Übersichtsarbeit den Kenntnisstand des präoperativen kognitiven Status als Voraussetzung für die Diagnose einer postoperativen kognitiven Dysfunktion (POCD, [16]). Die Erhebung des SIS vor der Intervention und wiederholt im Verlauf hilft bei der Einordnung einer postinterventionell bestehenden kognitiven Störung als vorbestehend, POCD oder Symptom eines Delirs. Auch Notaufnahmen anderer Disziplinen könnten über den SIS alltags- relevante Störungen der Kognition so sicher erfassen wie mit deutlich aufwendigeren Tests, die dort nicht durchführbar sind, ein kognitiver Einbruch sich somit in der Regel nicht über einen Punktverlust in der MMSE als Hinweis auf ein Delir [10] dokumentieren lässt. $\mathrm{Da}$ in der Erfassung und dem Monitoring eines Delirs die Fluktuation kognitiver Störung ein Leitsymptom darstellt [12], ist die engmaschige Wiederholbarkeit des SIS von diagnostischem Wert. Auch einige Hausärzte nutzen den SIS bereits für Verlaufskontrollen des kognitiven Status ihrer Patienten.

\section{Limitationen}

Da bei Patienten ohne Verdacht auf demenzielle Entwicklung gemäß Anamnese, klinischen Symptomen oder kognitivem Basis-Assessment keine aufwendigeren Verfahren zur Beurteilung der Kognition eingesetzt wurden, könnten Probanden trotz bereits eingetretener kognitiver Defizite während des Klinikaufenthalts unauffällig geblieben und somit das Arzturteil falsch-negativ ausgefallen sein.

Das Arzturteil als Referenzstandard wurde unter Berücksichtigung des gesamten klinischen Verlaufs gefällt; es konnte daher nicht für die Bestimmung der Änderungssensitivität herangezogen werden. Diese musste sich an weiteren kognitiven Tests orientieren, wobei nur 13 Studienteilnehmer gleichsinnige Veränderungen der Resultate aufwiesen. Die niedrige Fallzahl relativiert die Aussagefähigkeit zur Änderungssensitivität des SIS.

Die Durchführung der verschiedenen kognitiven Tests fand nicht verblindet statt, jedoch wurde der erste SIS, auf den wir uns für die Validität stützten, durch Ärzte durchgeführt, die an der weiteren Datenerhebung nicht beteiligt waren.

\section{Fazit für die Praxis}

- Der SIS ist bei geriatrischen Patienten in etwa 1 min ohne Material durchführbar und ideal geeignet für den Einsatz in der Aufnahmesituation.

- Er ist unabhängig von Sehstörungen und feinmotorischen Defiziten sowie auch bei reduzierter allgemeiner Belastbarkeit einsetzbar.

- Er korreliert gleich eng mit dem ärztlichen Gesamturteil wie die MMSE, deren Sensitivität und Spezifität er bei einem Grenzwert von 5 für den SIS und 25 für die MMSE übertrifft; auch die Korrelationen mit dem RWT und dem CDT belegen seine konvergente Validität.

- Alltagsrelevante kognitive Störungen sind bei Erreichen von weniger als 4 der 6 möglichen Punkte wahrscheinlich. Bei 5 Punkten sind sie mit hoher Wahrscheinlichkeit ausgeschlossen. Leichte kognitive Defizite sind erst bei voller Punktzahl weitgehend ausgeschlossen.

- Der Test kann bei zufriedenstellender Reliabilität unter Verwendung unterschiedlicher Worttripletts kurzfristig wiederholt werden.

\section{Korrespondenzadresse}

\section{Dr. S. Krupp}

Forschungsgruppe Geriatrie Lübeck, Krankenhaus Rotes Kreuz Lübeck Geriatriezentrum Marlistr. 10, 23566 Lübeck, Deutschland krupp@geriatrie-luebeck.de

\section{Einhaltung ethischer Richtlinien}

Interessenkonflikt. S. Krupp, A. Seebens, J. Kasper, M. Willkomm und F. Balck geben an, dass kein Interessenkonflikt besteht.

Für die Studie liegt ein positives Votum der Ethikkommission der Medizinischen Fakultät Dresden (EK $113032015)$ vor. Alle teilnehmenden Patienten haben ihr Einverständnis erklärt.

Open Access Dieser Artikel wird unter der Creative Commons Namensnennung 4.0 International Lizenz (http://creativecommons.org/licenses/by/4.0/deed. de) veröffentlicht, welche die Nutzung, Vervielfältigung, Bearbeitung, Verbreitung und Wiedergabe in jeglichem Medium und Format erlaubt, sofern Sie den/die ursprünglichen Autor(en) und die Quelle ordnungsgemäßnennen, einen Linkzur Creative Commons Lizenz beifügen und angeben, ob Änderungen vorgenommen wurden.

\section{Literatur}

1. Aschenbrenner A, Tucha O, Lange K (2000) RWT Regensburger Wortflüssigkeits-Test. Handanweisung. Hogrefe, Göttingen

2. Ashford JW (2008) Screening for memory disorders, dementia and Alzheimer's disease. Aging health 4:399-432 
3. Beck S, Büchi C, Lauber P, Grob D, Meier C (2014) Perioperative Risikostratifizierung geriatrischer Patienten bei nichtkardialen Eingriffen. Z Gerontol Geriatr 47:90-94

4. Beyermann S, Trippe RH, Bähr AA, Püllen R (2013) Mini-Mental-Status-Test im stationären geriatrischen Bereich. Eine Evaluation der diagnostischen Qualität.Z Gerontol Geriatr 46:740-747

5. Bowie P, Branton T, Holmes J (1999) Should the mini mental state examination be used to monitor dementia treatments? Lancet 354:1527-1528

6. Callahan CM, Unverzagt FW, Hui SL, Perkins AJ, Hendrie HC (2002) Six-item screener to identify cognitive impairment among potential subjects for clinical research. Med Care 40:771-778

7. Carpenter CR, DesPain B, Keeling TN, Shah M, Rothenberger M (2011) The Six-Item Screener and AD8 for the detection of cognitive impairment in geriatric emergency department patients. Ann Emerg Med 57:653-661

8. Fillenbaum GG, Wilkinson WE, Welsh KA, Mohs RC (1994) Discrimination between stages of Alzheimer's disease with subsets of Mini-Mental State Examination items. An analysis of Consortium to Establish a Registry for Alzheimer's Disease data. Arch Neurol 51:916-921

9. Folstein MF, Folstein SE, McHugh PR (1975) “Minimental state". A practical method for grading the cognitive state of patients for the clinician. J Psychiatr Res 12:189-198

10. Frühwald $T$, Weissenberger-Leduc $M$, Jagsch $C$, Singler K, Gurlit S, Hofmann W, Böhmdorfer
B, Iglseder B (2014) Delir. Eine interdisziplinäre Herausforderung. Z Gerontol Geriatr 47:425-440

11. Kessler J, Markowitsch HJ, Denzer P (1990) MiniMental-Status-Test von M. F. Folstein, S. E. Folstein und P. R. McHugh (deutschsprachige Fassung). Beltz, Weinheim

12. Lorenzl S, Füsgen I, Noachtar S (2012) Verwirrtheitszustände im Alter - Diagnostik und Therapie Dtsch Arztebl Int 109:391-400

13. Montgomery SA, Asberg M (1979) A new depression scale designed to be sensitive to change. Br JPsychiatry 134:382-389

14. Ramlall S, Chipps J, Bhigjee Al, Pillay BJ (2013) The sensitivity and specificity of subjective memory complaints and the subjective memory rating scale, deterioration cognitive observee, minimental state examination, six-item screener and clock drawing test in dementia screening. Dement Geriatr Cogn Disord 36:119-135

15. Rösler $M$, Frey $U$, Retz-Junginger $P$, Supprian T, Retz W (2003) Diagnostik in der Demenz: Standardisierte Untersuchungsinstrumente im Überblick. Fortschr Neurol Psychiatr 71:187-198

16. Rundshagen I (2014) Postoperative cognitive dysfunction. Dtsch Arztebl Int 111(8):119-125

17. Sampson EL, Blanchard MR, Jones L, Tookman A King M (2009) Dementia in the acute hospital: prospective cohort study of prevalence and mortality. Br J Psychiatry 195:61-66

18. Shulman Kl, Shedletsky R, Silver IL (1986) The challenge of time: clock-drawing and cognitive function in the elderly. Int J Geriatr Psychiatry 1:135-140
19. Shulman KI, GoldDP, Cohen CA,ZuccheroCA(1993) Clock drawing and dementia in the community: a longitudinal study. Int J Geriatr Psychiatry 8:487-496

20. Wilber ST, Carpenter CR, Hustey FM (2008) The Six-Item Screener to detect cognitive impairment in older emergency department patients. Acad Emerg Med 15:613-616

21. Wolter DK (2014) Beginnende Demenz und Fahreignung. Teil 2: Das Assessment und seine praktischen Konsequenzen. Z Gerontol Geriatr 47:345-355

\section{Springer Medizin}

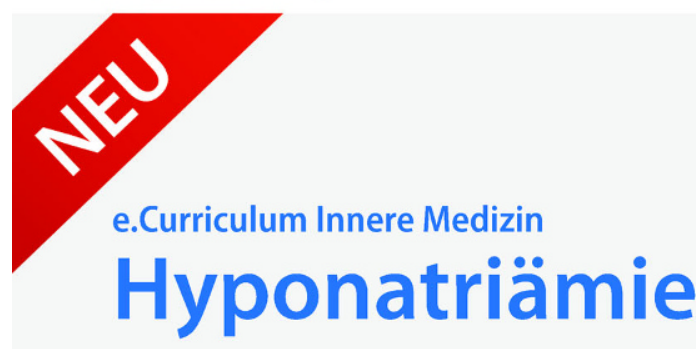

\section{E-Learning für Internisten}

\author{
- Nach der aktuellen Leitlinie \\ - Fallbasiertes E-Learning \\ - Zertifiziert mit 3 Punkten \\ - Exklusiv für DGIM-Mitglieder und \\ e.Med-Abonnenten
}

Deutsche Gesellschaft für Innere Medizin e.V.

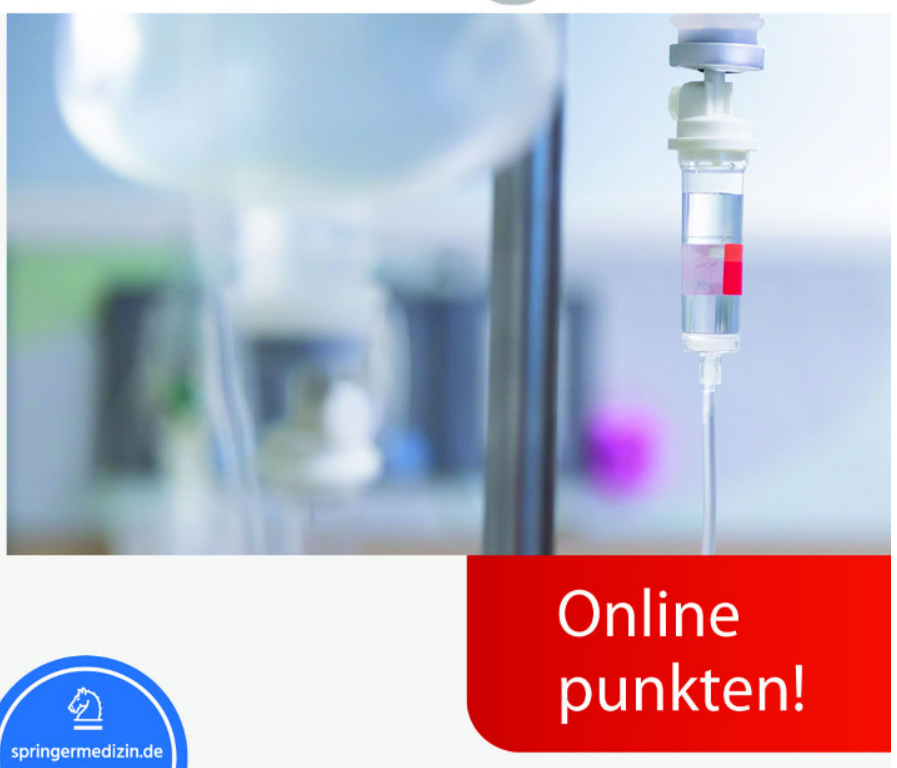

